\title{
Can stress-strain relationships be obtained from indentation curves using conical and pyramidal indenters?
}

\author{
Yang-Tse Cheng ${ }^{\text {a) }}$ \\ Materials and Processes Laboratory, General Motors Research and Development Center, \\ Warren, Michigan 48090 \\ Che-Min Cheng ${ }^{\mathrm{b})}$ \\ Laboratory for Non-Linear Mechanics of Continuous Media, Institute of Mechanics, \\ Chinese Academy of Sciences, Beijing 100080, China
}

(Received 8 February 1999; accepted 28 June 1999)

\begin{abstract}
Applying the scaling relationships developed recently for conical indentation in elastic-plastic solids with work-hardening, we examine the question of whether stress-strain relationships of such solids can be uniquely determined by matching the calculated loading and unloading curves with that measured experimentally. We show that there can be multiple stress-strain curves for a given set of loading and unloading curves. Consequently, stress-strain relationships may not be uniquely determined from loading and unloading curves alone using a conical or pyramidal indenter.
\end{abstract}

For nearly 100 years, indentation experiments have been performed to obtain the hardness of materials. ${ }^{1} \mathrm{Re}-$ cent years have seen significant improvements in indentation equipment and a growing need for measuring the mechanical properties of materials on small scales. ${ }^{2,3}$ With these improvements, it is now possible to monitor, with high precision and accuracy, both the load and displacement of an indenter during indentation experiments. From the loading-unloading curves, the hardness and elastic modulus of materials can be obtained using the methods proposed by Doerner and Nix, ${ }^{4}$ Oliver and Pharr, ${ }^{5}$ or Cheng and Cheng. ${ }^{6}$ Recently, a number of papers have also suggested the possibility of extracting the mechanical properties of materials by matching the loading and unloading curves calculated using finite element methods with that measured experimentally. ${ }^{7-9}$ However, the question remains as to whether the stressstrain relationships can be uniquely determined from the loading-unloading curves alone.

In this paper, we first examine the essential features of indentation loading and unloading curves using the scaling relationships for conical indentation in elastic-plastic solids. ${ }^{10,11}$ Using these features, we show that essentially the same loading-unloading curves can be constructed form different stress-strain relationships. Consequently, stress-strain relationships may not be uniquely determined from loading and unloading curves alone using a conical or pyramidal indenter.

\footnotetext{
a) Address all correspondence to this author.

e-mail: Yang_T._Cheng@ @otes.gmr.com

b)e-mail: zhengzm@LNM.imech.ar.cn
}

We consider a three dimensional, frictionless, rigid conical indenter of a given half angle, $\theta$, indenting normally into an elastic-plastic solid with work-hardening. The stress-strain $(\sigma-\varepsilon)$ curves of the solids under uniaxial tension are assumed to be given by

$$
\begin{array}{ll}
\sigma=E \varepsilon \quad, & \text { for } \varepsilon \leq \frac{Y}{E}, \\
\sigma=K \varepsilon^{n}, & \text { for } \varepsilon \geq \frac{Y}{E},
\end{array}
$$

where $E$ is Young's modulus, $Y$ is initial yield stress, $K$ is strength coefficient, and $n$ is work-hardening exponent. ${ }^{12}$ To ensure continuity, we note $K=Y[E / Y]^{n}$. Consequently, $E, Y, n$, and Poisson's ratio, $v$, are sufficient to describe the stress-strain relationship. When $n$ is zero, Eq. (1) becomes the model for elastic-perfectly plastic solids. For most metals $n$ has a value between 0.1 and $0.5 .^{13}$

From geometric self-similarity, the equations describing the relationships between force, $F$, and indenter displacement, $h$, can be written as

$$
\begin{aligned}
& F=E h^{2} \Pi_{\alpha}\left(\frac{Y}{E}, v, n, \theta\right), \text { for loading, } \\
& F=E h^{2} \Pi_{\gamma}\left(\frac{Y}{E}, \frac{h}{h_{m}}, v, n, \theta\right) \quad, \text { for unloading, }
\end{aligned}
$$

where $\Pi_{\alpha}$ and $\Pi_{\gamma}$ are two dimensionless functions, and $h_{m}$ is the maximum depth the indenter reaches immediately before unloading.

(C) 1999 Materials Research Society

3493 
From these equations, the essential features of loadingunloading curves can be obtained. For example, the initial unloading slope, $d F /\left.d h\right|_{h=h_{m}}$, is given by

$$
\begin{aligned}
\left.\frac{1}{E h_{m}} \frac{d F}{d h}\right|_{h=h_{m}} & =\Pi_{\gamma}^{\prime}\left(\frac{Y}{E}, 1, \nu, n, \theta\right)+2 \Pi_{\gamma}\left(\frac{Y}{E}, 1, \nu, n, \theta\right) \\
& \equiv \Pi_{\delta}\left(\frac{Y}{E}, \nu, n, \theta\right)
\end{aligned}
$$

where $\Pi_{\delta}$ is a dimensionless function of $Y / E, \nu, n$, and $\theta$. The final depth, $h_{f}$, at which the force on the indenter reaches zero during unloading is a solution of Eq. $\left(2^{\prime}\right)$ for $F=0$ and can, therefore, be written as

$$
\frac{h_{f}}{h_{m}}=\Pi_{\phi}\left(\frac{Y}{E}, \nu, n, \theta\right),
$$

where $\Pi_{\phi}$ is a dimensionless function.

From Eq. (2), the total work done by the indenter, $W_{t o t}$, to cause elastic and plastic deformation when the indenter reaches the maximum depth, $h_{m}$, is given by

$$
W_{t o t}=\int_{0}^{h_{m}} F d h=\frac{E h_{m}^{3}}{3} \Pi_{\alpha}\left(\frac{Y}{E}, \nu, n, \theta\right) .
$$

The work done by the solid to the indenter during unloading, $W_{w}$, can be expressed, using Eq. (2'), as

$$
W_{u}=\int_{h_{f}}^{h_{m}} F d h=E h_{m}{ }^{3} \int_{\frac{h_{f}}{h_{m}}}^{1} x^{2} \Pi_{\gamma}\left(\frac{Y}{E}, x, \nu, n, \theta\right) d x .
$$

Using Eq. (4), the integral in Eq. (6) is independent of displacement. Consequently, the ratio of $\left(W_{t o t}-W_{u}\right) / W_{t o t}$ is independent of $h_{m}$,

$$
\frac{W_{t o t}-W_{u}}{W_{t o t}}=\Pi_{\omega}\left(\frac{Y}{E}, v, n, \theta\right)
$$

i.e., a dimensionless function of $Y / E, v, n$, and $\theta$.

The dimensionless functions $\Pi_{i}(Y / E, v, n, \theta)(i=$ $a, \delta, \phi, \omega)$ have been calculated using ABAQUS ${ }^{14}$ finite element analysis. The finite element model has been discussed in detail previously. ${ }^{10,11,15}$ The rate-independent, incremental theory of plasticity in ABAQUS was used for the finite element calculations. In particular, the plasticity theory uses the Mises yield surface model with associated plastic flow rule. The hardening rule used was that of isotropic hardening and the hardening curves were given by Eq. (1). The frequently used half angle of $68^{\circ}$ for the rigid indenter and a typical Poisson's ratio of 0.3 for the solid are chosen to illustrate the essential physics of conical indentation in elastic-plastic solids with workhardening. To simplify notation, $\Pi_{i}(Y / E, n)(i=a, \delta, \phi, \omega)$ is used instead of $\Pi_{i}\left(Y / E, 0.3, n, 68^{\circ}\right)(i=a, \delta, \phi, \omega)$. These scaling functions are shown in Figs. 1(a) to 1(d).

An inspection of Figs. 1(c) and 1(d) suggests that the two quantities, $\Pi_{\phi}(Y / E, n)=h_{f} / h$ and $\Pi_{\omega}(Y / E, n)=$ $\left(W_{t o t}-W_{u}\right) / W_{t o t}$, are strongly correlated within our range of calculations. An approximately linear dependence between the two is shown in Fig. 2. In fact, Loubet et al. ${ }^{16}$ was the first to suggest, based on experimental observations, a linear relationship between $\left(W_{t o t}-W_{u}\right) / W_{t o t}$ and
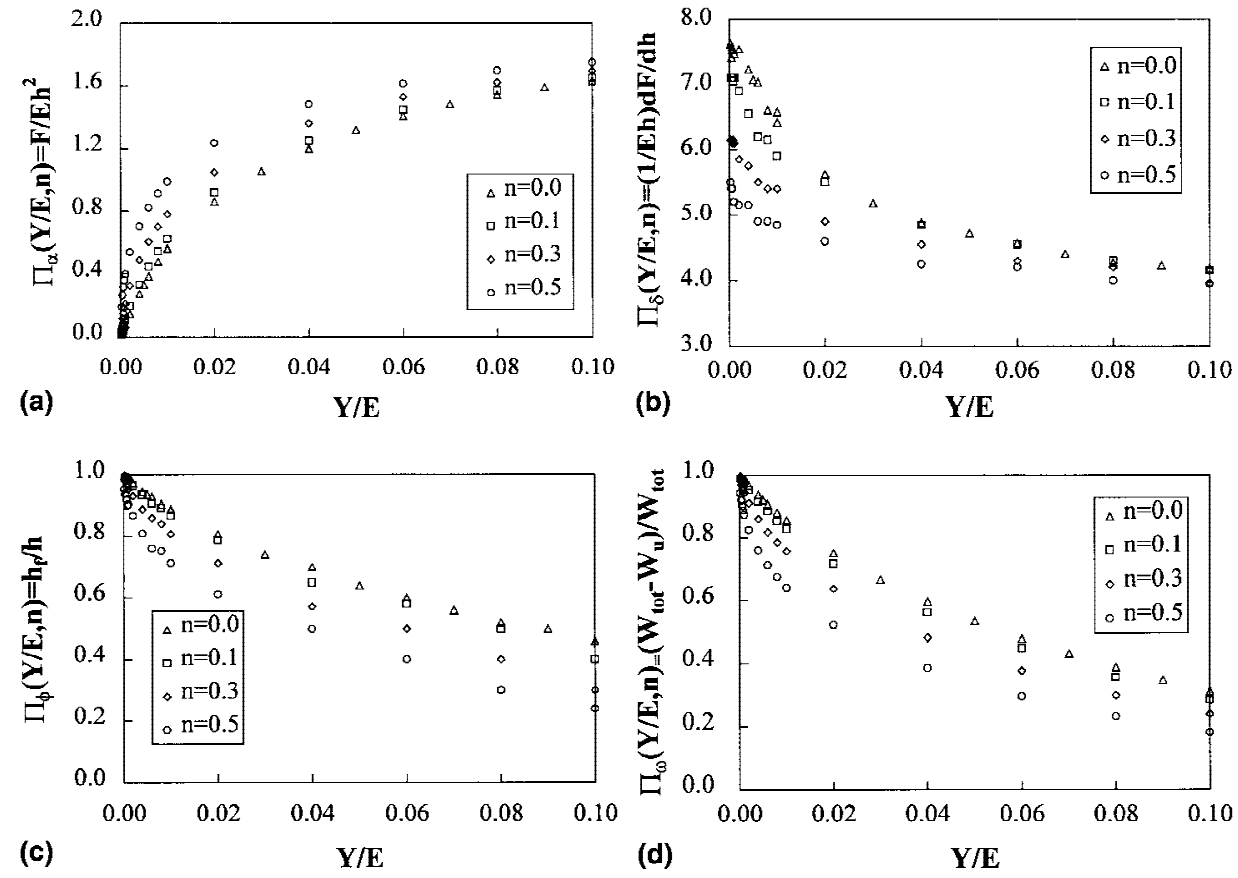

FIG. 1. Scaling relationships for (a) $F / E h^{2}$, (b) $(1 / E h) d F / d h$, (c) $h_{f} / h$, and (d) $\left(W_{t o t}-W_{u}\right) / W_{t o t}$. 
$h_{f} / h$. The present extensive finite element results (Fig. 2) corroborate their empirical observation. Consequently, Eqs. (2), (3), and (4) [or (7)] and the respective Figs. 1(a), 1(b), and 1(c) [or 1(d)] can be used to describe the essential features of indentation loading and unloading curves.

For a given set of basic mechanical properties, $E, v, Y$, and $n$, the essential features such as the loading curve, $d F /\left.d h\right|_{\mathrm{h}=\mathrm{h}_{\mathrm{m}}}$, and $h_{f} / h_{m}$ [or $\left.\left(W_{t o t}-W_{u}\right) / W_{t o t}\right]$ can be determined from Eqs. (2), (3), and (4) [or (7)]. Conversely, these scaling functions can be used to find the appropriate values of $E, v, Y$, and $n$ for a known set of essential features. However, this inverse problem is not unique.

The non-uniqueness is demonstrated as follows. For a given $v$, the Young's modulus, $E$, can be uniquely determined from loading-unloading curves using, for example, the method of Cheng and Cheng. ${ }^{6}$ Now with known $E$ and $\nu$, we first find values of $Y$ and $n$ that result in the same loading curves. This is accomplished by drawing a straight line in Fig. 1(a) parallel to the horizontal axis (i.e., the $Y / E$ axis). Those values of $Y$ and $n$ corresponding to the intersects of the horizontal line and $\Pi_{\alpha}(Y / E, n)$ lead to the same loading curves. Similarly, the values of $Y$ and $n$ that generate the same initial unloading slopes and final depths can be obtained from Figs. 1(b) and 1(c). In fact, the range of values of $Y$ and $n$ found from Figs. 1(a)-1(c) overlaps with each other. Consequently, the values of $Y$ and $n$ that satisfy all three conditions in Figs. 1(a)-1(c) can be found. Following this procedure, two sets of essentially the same loading and unloading curves calculated using ABAQUS are shown in Figs. 3(a) and 3(b) for the cases of large and small $Y / E$, respectively. It is evident that the loading and unloading curves are indistinguishable if the essential features such as the initial unloading slope, final depth, and maximum load are the same. Therefore, loading and unloading curves from conical indentation alone cannot uniquely determine stress-strain relationships.

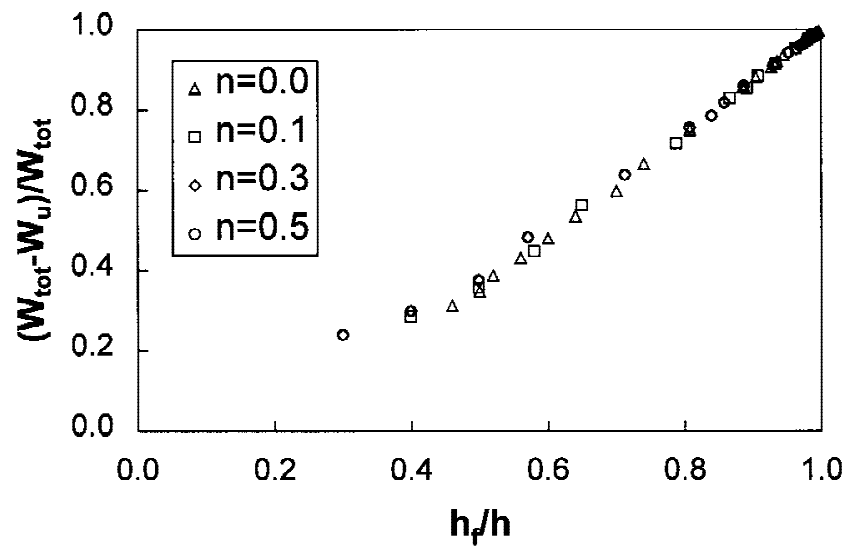

FIG. 2. A relationship between $\left(W_{\text {tot }}-W_{u}\right) / W_{\text {tot }}$ and $h_{f} / h$ for $0<h_{f} / h<1.0$.
Because pyramidal indenters are also geometrically self-similar, scaling relationships such as those for conical indenters exist. ${ }^{11}$ Furthermore, because previous numerical work has shown that the loading and unloading curves are the same for pyramidal and conical indentation, provided that the volume-to-depth relationships are the same for the two types of indenters. ${ }^{17}$ Consequently, loading and unloading curves from pyramidal indentation alone also cannot uniquely determine stress-strain relationships.

We have shown that the stress-strain relationships may not be uniquely determined from indentation loading and unloading curves obtained using a conical or pyramidal indenter. Hardness and elastic modulus, however, can be obtained from these curves alone. ${ }^{4-6} \mathrm{Al}-$ though the above conclusions were obtained for ideally sharp conical and pyramidal indenters, they should be applicable to those indenters with rounded tips, provided that the indentation depth is sufficiently large and the
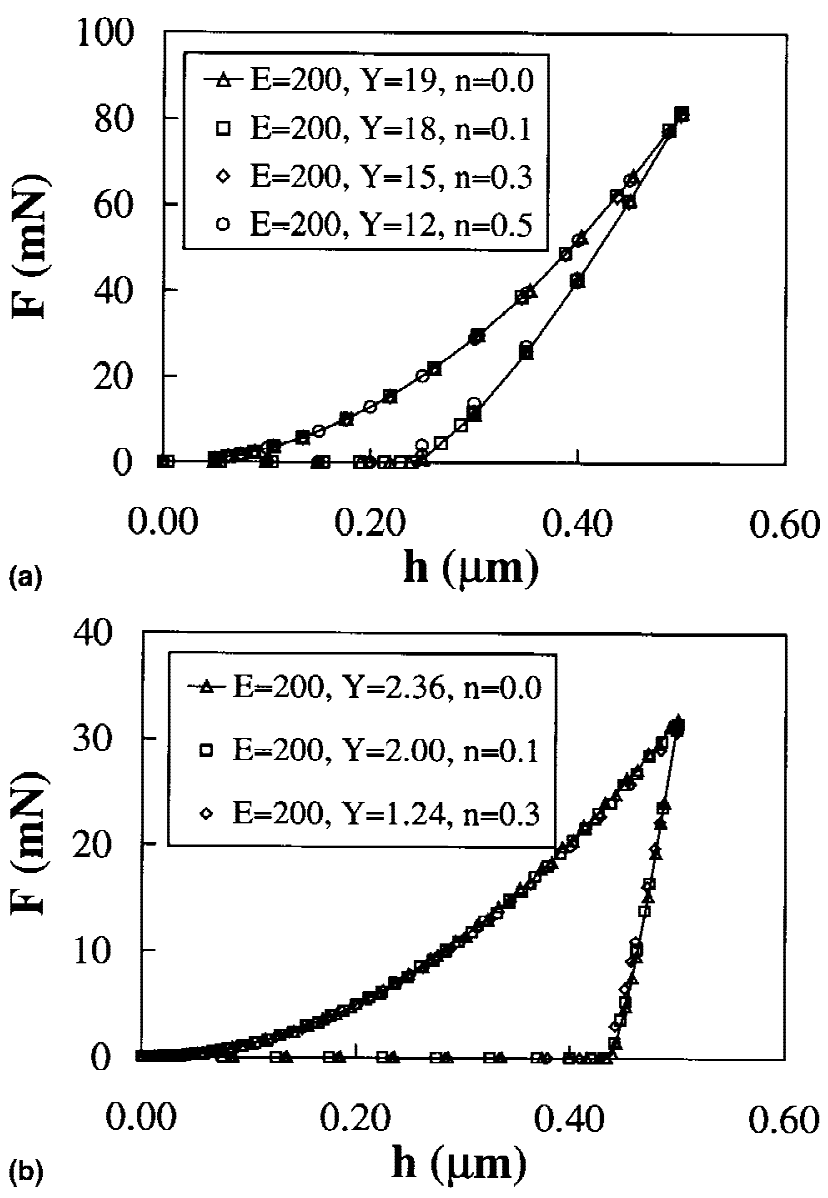

FIG. 3. Examples of overlapping loading and unloading curves for (a) highly elastic solids (i.e., large Y/E) and for highly plastic solids (b) (i.e., small Y/E). The Poisson's ratio is 0.3 for these cases. The calculated hardness values are (a) 31.7, 32.6, 31.4, and 32.1 GPa for the respective four cases $(\triangle, \square, \diamond$, and $\bigcirc)$ and (b) 6.0, 6.5, and 7.4 GPa for the respective three cases $(\triangle, \square$, and $\diamond)$. 
loading and unloading curves approach those obtained using the ideally sharp indenters. A discussion on the effects of tip rounding on the shape of indentation loading curves has recently appeared. ${ }^{18}$ When the indentation depth is small or when spherical indenters are used, the question of whether stress-strain relationships of solids can be uniquely determined by matching the calculated loading and unloading curves with the measured ones remains to be investigated. The possibilities of using several conical indenters of different angles to obtain stressstrain relationships should also be investigated both experimentally and theoretically.

\section{ACKNOWLEDGMENTS}

We would like to thank W.J. Meng, J.R. Smith, W. Yang, S.J. Harris, G.L. Eesley, L.C. Lev, B. Qiu, S.F. Yin, D.D. Snyder, and K.C. Taylor for helpful discussions. We would also like to thank R.J. Blint, M.S. Meyer, K. Ueno, J. Brown (EDS), P. Lalor (HKS), and L. Hill (HKS) for maintaining the workstations and for helping with the use of ABAQUS.

\section{REFERENCES}

1. D. Tabor, The Hardness of Metals (Oxford, London, 1951).

2. J.B. Pethica, R. Hutchings, and W.C. Oliver, Phil. Mag. A48, 593 (1983).
3. D. Stone, W.R. LaFontaine, P. Alexopoulos, T.W. Wu, and C-Y. Li, J. Mater. Res. 3, 141 (1988).

4. M.F. Doener and W.D. Nix, J. Mater. Res. 1, 601 (1986).

5. W.C. Oliver and G.M. Pharr, J. Mater. Res. 7, 1564 (1992).

6. Y-T. Cheng and C-M. Cheng, Appl. Phys. Lett. 73, 614 (1998).

7. A.K. Bhattacharya, and W.D. Nix, Int. J. Solids Structures 24, 881 (1988).

8. T.A. Laursen, and J.C. Simo, J. Mater. Res. 7, 618 (1992).

9. S.M. Myers, J.A. Knapp, D.M. Follstaedt. and M.T. Dugger, J. Appl. Phys. 83, 1256 (1998).

10. Y-T. Cheng and C-M. Cheng, Int. J. Solids Structures 36, 1231 (1999).

11. Y-T. Cheng and C-M. Cheng, J. Appl. Phys. 84, 1284 (1998).

12. J. Lubliner, Plasticity Theory (Macmillan, New York, 1990).

13. G. Dieter, Mechanical Metallurgy, Second Edition (McGraw-Hill, New York, 1976).

14. ABAQUS, version 5.6 and 5.7, Hibbitt, Karlsson \& Sorensen, Inc. (Pawtucket, RI 02860, USA).

15. Y-T. Cheng and C-M. Cheng, Mag. Lett. 77, 39 (1998).

16. J.L. Loubet, J.M. Georges, O. Marchesini, and G. Meille, Trans. Am. Soc. Mech. Eng.: J. Tribology 106, 43 (1984).

17. M. Lichinchi, C. Lenardi, J. Haupt, and R. Vitali, Thin Solid Films 312, 240 (1998).

18. Y-T. Cheng and C-M. Cheng, J. Mater. Res. 13, 1059 (1998). 